\title{
Diagnostic value and lymph node metastasis prediction of a custom-made panel (thyroline) in thyroid cancer
}

\author{
ZUNFU KE ${ }^{1,2^{*}}$, YIHAO LIU ${ }^{1,5^{*}}$, YUNJIAN ZHANG ${ }^{3 *}, \mathrm{JIE} \mathrm{LI}^{3}$, MING KUANG $^{4}$, \\ SUI PENG ${ }^{5}$, JINYU LIANG ${ }^{6}$, SHUANG YU $^{1}$, LEI SU $^{1}$, LILI CHEN $^{2}$, CONG SUN $^{2}$, \\ $\mathrm{BIN} \mathrm{LI}^{5}$, JESSICA CAO ${ }^{7}$, WEIMING $\mathrm{LV}^{3}$ and HAIPENG XIAO ${ }^{1}$

\begin{abstract}
Departments of ${ }^{1}$ Endocrinology, ${ }^{2}$ Pathology and ${ }^{3}$ Breast and Thyroid Surgery; ${ }^{4}$ Cancer Center;
${ }^{5}$ Clinical Trial Unit; ${ }^{6}$ Department of Ultrasound, The First Affiliated Hospital of Sun Yat-sen University,

Guangzhou, Guangdong 510080, P.R. China; ${ }^{7}$ Faculty of Medicine, University of Toronto, Toronto, ON M5S 1A8, Canada
\end{abstract}

Received February 12, 2018; Accepted June 5, 2018

DOI: $10.3892 / o r .2018 .6493$

\begin{abstract}
Differentiation of benign and malignant thyroid nodules is crucial for clinical management. Here, we explored the efficacy of next-generation sequencing (NGS) in predicting the classification of benign and malignant thyroid nodules and lymph node metastasis status, and simultaneously compared the results with ultrasound (US). Thyroline was designed to detect 15 target gene mutations and 2 fusions in 98 formalin-fixed, paraffin-embedded (FFPE) tissues, including those from 82 thyroid cancer (TC) patients and 16 patients with benign nodules. BRAF mutations were found in $57.69 \%$ of the papillary thyroid cancer (PTC) cases, while RET mutations were detected among all the medullary thyroid cancer (MTC) cases. Multiple mutations were positive but none showed dominance in anaplastic thyroid cancer (ATC) and follicular thyroid cancer (FTC). The sensitivity and specificity of NGS prediction in differentiation of benign and malignant thyroid nodules were 79.27 and $93.75 \%$, respectively, and the positive predictive value (PPV) and negative predictive value (NPV) were 98.48 and $46.88 \%$, respectively. The sensitivity and
\end{abstract}

Correspondence to: Dr Haipeng Xiao, Department of Endocrinology, The First Affiliated Hospital of Sun Yat-sen University, 58 ZhongShan Second Road, Guangzhou, Guangdong 510080, P.R. China

E-mail: xiaohp@mail.sysu.edu.cn

*Contributed equally

Abbreviations: TC, thyroid cancer; US, ultrasound; FNA, fine-needle aspiration; NGS, next-generation sequencing; FFPE, formalin-fixed, paraffin-embedded; TI-RADS, Thyroid Imaging Reporting and Data System; PPV, positive predictive value; NPV, negative predictive value; PTC, papillary thyroid cancer; ATC, anaplastic thyroid cancer; MTC, medullary thyroid cancer; FTC, follicular thyroid cancer; ROC, receiver operator characteristic; AUC, area under curve

Key words: thyroid cancer, next-generation sequencing, Thyroid Imaging Reporting and Data System, accurate diagnosis, metastasis specificity of US were 76.83 and $6.25 \%$, respectively, and the PPV and NPV were 80.77 and $5.00 \%$, respectively. The area under curve (AUC) of NGS and US were 0.865 and 0.415 , respectively. A total of 27 patients had $\geq 1$ metastases to lymph nodes, 19 of which carried mutations, including BRAF, RET, NRAS, PIK3CA, TP53, CTNNB1 and PTEN. However, there was no correlation between the variant allele frequency of specific gene mutations and the number of metastatic lymph nodes. In conclusion, the prediction value of NGS was higher than the US-based Thyroid Imaging Reporting and Data System (TI-RADS). NGS is valuable for the accurate differentiation of benign and malignant thyroid nodules, and pathological subtypes in FFPE samples. The findings of the present study may pave the way for the application of NGS in analyzing fine-needle aspiration (FNA) biopsy samples.

\section{Introduction}

Thyroid cancer (TC) is the most common endocrine tumor and its incidence has continually increased over recent decades (1-5). Thyroid nodules are found in $50-60 \%$ of the population in adulthood, while only $5 \%$ of thyroid nodules are malignant (6). A previous study found that preoperative image examination and operation process missed $33 \%$ of lymph node metastases in TC patients (7). As a result, the main challenge in the management of thyroid nodules is to rule out malignancy from massive thyroid nodules and predict lymph node metastasis.

The diagnosis of TC is typically obtained through ultrasound (US) examination and fine-needle aspiration (FNA) biopsy (8). However, current US has an accuracy of only $72.6 \%$ (9). Between 20 and 30\% of FNA biopsy samples may draw incorrect or indeterminate conclusions based on simple cytology $(10,11)$. The current predictive value of US and FNA cannot fully meet the requirements of accurate diagnosis of TC. Accurate identification of the pathological subtype and lymph node metastasis status of TC preoperatively is important in developing individualized therapeutic strategies.

Genetic examination, as a newly-developed technique for cancer diagnosis, has been demonstrated to have advantages in the early diagnosis of cancer and in accelerating the examination process $(12,13)$. As the most common gene mutation found 
in TC, BRAF plays an important role in TC diagnosis and risk stratification (12). With the introduction of next-generation sequencing (NGS), high throughput sequencing is able to detect target mutations using only a small amount of DNA. The detection of mutations in TC is useful for providing a specific diagnosis in cytologically indeterminate cases $(12,14)$.

In this study, we focused on the application of NGS for a panel of mutations in TC and evaluated its predictive efficacy in classifying benign and malignant nodules and their metastasis status, compared with conventional US examination and histological diagnosis.

\section{Materials and methods}

Thyroid samples. A total of 98 formalin-fixed, paraffin-embedded (FFPE) tissue specimens from surgically removed thyroid samples were collected at the Department of Pathology of the First Affiliated Hospital of Sun Yat-sen University between 2011 and 2016. Subsequent analyses were performed on 16 thyroid benign nodules and 82 TC specimens. Based on histological results, all tumors were classified by two independent pathologists. All patients provided informed consent before enrollment in the study, which was approved by the Ethics Committee of the First Affiliated Hospital of Sun Yat-sen University.

Tissue DNA extraction. DNA was extracted using QIAamp DNA FFPE tissue kit (Qiagen, Inc., Valencia, CA, USA) according to the manufacturer's instructions. DNA concentration was measured using a Qubit dsDNA assay.

NGS library preparation. DNA shearing was performed using Covaris M220, followed by end repair, phosphorylation and adaptor ligation. Fragments of 200-400 bp in size were selected by beads (Agencourt AMPure XP kit; Beckman Coulter, Inc., Brea, CA, USA), followed by hybridization with capture probe baits, hybrid selection with magnetic beads and PCR amplification. A bioanalyzer high-sensitivity DNA assay was performed to assess the quality and size of the fragments. Indexed samples were sequenced on a NextSeq 500 sequencer (Illumina, Inc., San Diego, CA, USA) with pair-end reads.

Capture-based targeted DNA sequencing. Genetic profiles of all tissue samples were assessed by capture-based targeted deep sequencing, using a lymphoma panel consisting of critical exons and introns of 31 genes (Burning Rock Biotech, Ltd., Guangzhou, China), covering $209 \mathrm{~kb}$ of human genomic regions. DNA quality and size were assessed by high sensitivity DNA assay using a bioanalyzer. All indexed samples were sequenced on a NextSeq 500 (Illumina, Inc.) with pair-end reads. When the NGS analysis was performed in thyroid-derived tumor samples, the white blood cells (WBCs) from the same patient were used as their own inner controls to ensure that the detected mutations were definitely somatic.

Sequence data analysis. After a successful sequencing reaction, the raw sequence data were mapped to the human genome (hg19) using BWA Aligner 0.7.10. Local alignment optimization, variant calling and annotation were performed using GATK 3.2, MuTect and VarScan. Variants were filtered
Table I. Demographic and nodule characteristics.

\begin{tabular}{|c|c|c|}
\hline & \multicolumn{2}{|c|}{ Histological diagnosis } \\
\hline & $\begin{array}{l}\text { Benign group } \\
\qquad(\mathrm{n}=16)\end{array}$ & $\begin{array}{l}\text { Malignant group } \\
\qquad(\mathrm{n}=82)\end{array}$ \\
\hline \multicolumn{3}{|l|}{ Patient information } \\
\hline Age (years) & $48.00 \pm 6.07$ & $45.95 \pm 3.20$ \\
\hline Sex ratio $(M / F)$ & $6 / 10$ & $25 / 57$ \\
\hline \multicolumn{3}{|l|}{ Nodule characteristic } \\
\hline Unique/multinodular & $16 / 0$ & $73 / 9$ \\
\hline $\begin{array}{l}\text { Maximun size } \\
(\mathrm{mm} ; \text { mean } \pm \mathrm{SD})\end{array}$ & $7.3 \pm 6.1$ & $13.7 \pm 11.6$ \\
\hline \multicolumn{3}{|l|}{ Echo genicity } \\
\hline Hypoechoic & 8 & 39 \\
\hline Isoechoic & 3 & 12 \\
\hline Hyperechoic & 2 & 10 \\
\hline Unknown & 3 & 21 \\
\hline \multicolumn{3}{|l|}{ Blood flow } \\
\hline Rich & 9 & 35 \\
\hline Normal & 1 & 0 \\
\hline Low & 4 & 22 \\
\hline Unknown & 2 & 25 \\
\hline \multicolumn{3}{|l|}{ Microcalcification } \\
\hline Positive & 9 & 38 \\
\hline Negative & 5 & 15 \\
\hline Unknown & 2 & 29 \\
\hline \multicolumn{3}{|l|}{ Aspect ratio } \\
\hline$<1$ & 11 & 46 \\
\hline$>1$ & 4 & 5 \\
\hline Unknown & 1 & 24 \\
\hline \multicolumn{3}{|l|}{ Boundary } \\
\hline Clear & 7 & 33 \\
\hline Unclear & 6 & 18 \\
\hline Unknown & 3 & 31 \\
\hline TI-RADS category 4 & $15 / 16$ & $63 / 82$ \\
\hline \multicolumn{3}{|l|}{ Pathological subtype } \\
\hline PTC & - & 52 \\
\hline ATC & - & 13 \\
\hline MTC & - & 10 \\
\hline FTC & - & 7 \\
\hline \multicolumn{3}{|l|}{ Lymph node metastasis } \\
\hline Positive & - & 27 \\
\hline Negative & - & 55 \\
\hline
\end{tabular}

SD, standard deviation; TI-RADS, Thyroid Imaging Reporting and Data System; PTC, papillary thyroid cancer; ATC, anaplastic thyroid cancer; MTC, medullary thyroid cancer; FTC, follicular thyroid cancer.

using the VarScan fpfilter pipeline. At least 5 supporting reads were required for INDELs, while 8 supporting reads were required for SNVs to be defined. According to the ExAC, 1000 Genomes, dbSNP and ESP6500SI-V2 databases, 
A

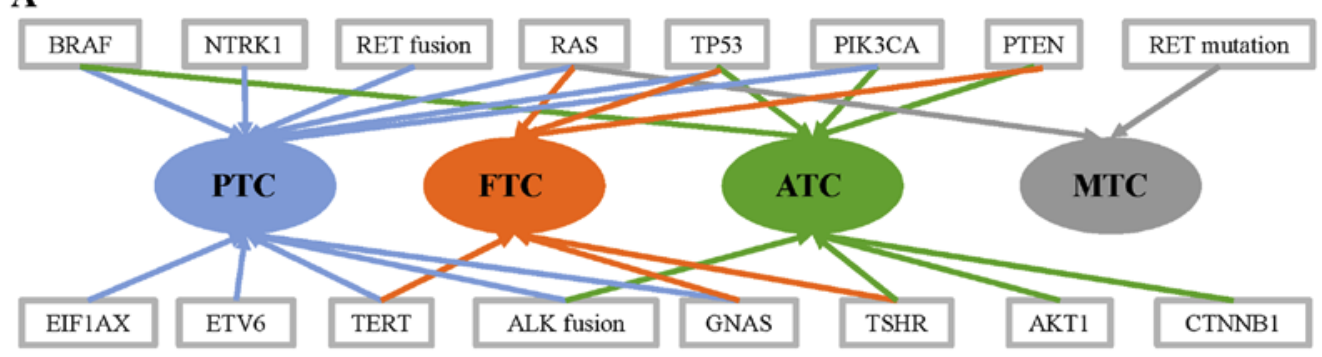

B
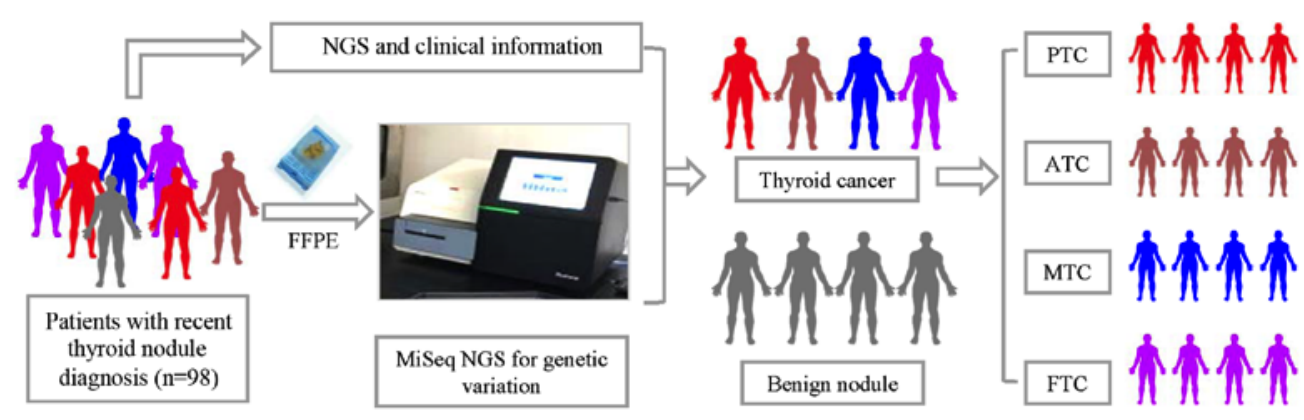

C
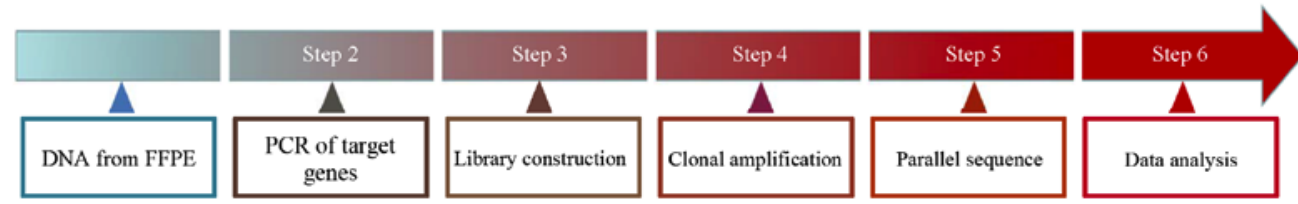

Figure 1. Gene mutations and fusions in subtypes of TC and workflow of NGS. (A) BRAF, RAS, TERT, ETV6, EIF1AX, GNAS, PIK3CA, TP53 and NTRK1 mutations, as well as RET and ALK fusions, were found in PTC. BRAF, TERT, ALK fusion, GNAS, AKT1, PIK3CA, TP53 and PTEN were found in ATC. RAS, TERT, TSHR, GNAS, PENT and TP53 were found in FTC, while only RET and RAS mutations were found in MTC. (B) FFPE samples were obtained from 98 thyroid nodule patients, which was followed by CTC enumeration on NanoVelcro Chips. After collecting clinical information, we analyzed the correlation between pathological information and NGS results. (C) DNA from FFPE tissue was amplified for enrichment of target regions in a multiplex PCR reaction. Then, the library was prepared by ligating the PCR amplicons into platform-specific adapters and adding bar codes for specimen multiplexing. Finally, the library was enriched by clonal amplification (emPCR) and sequenced by massively parallel sequencing on the Ion Torrent PGM. The data analysis and variant calling were performed using bioinformatic pipelines followed by a custom SeqReporter algorithm for filtering and annotation of genetic variants. TC, thyroid cancer; NGS, next-generation sequencing; PTC, papillary thyroid cancer; ATC, anaplastic thyroid cancer; FTC, follicular thyroid cancer; MTC, medullary thyroid cancer; FFPE, formalin-fixed, paraffin-embedded.

variants with population frequency $>0.1 \%$ were grouped as SNPs and excluded from further analysis. Remaining variants were annotated with ANNOVAR and SnpEff v3.6. DNA translocation analysis was performed using both TopHat 2 and Factera 1.4.3.

Thyroid Imaging Reporting and Data System (TI-RADS) category. According to TI-RADS (15), thyroid nodules classified as category 4 with at least one suspicious US feature are believed to have a high probability of malignancy. The following US features showed a significant association with malignancy: solid component, hypoechogenicity, marked hypoechogenicity, microlobulated or irregular margin, microcalcification and taller-than-wide shape. Thyroid nodules without the above features were classified as category $<4$ (low risk).

Statistical analysis. Calculations of specificity, sensitivity, positive predictive value (PPV), negative predictive value (NPV) and receiver operator characteristic (ROC) were conducted using IBM SPSS 16.0 for Windows (SPSS, Inc., Chicago, IL, USA). Correlations were studied using Spearman's rank correlation coefficients. Significance level was set as $\alpha=0.05$.

\section{Results}

Thyroline NGS panel design. To design a NGS sequencing panel for thyroid nodule patients, we conducted a review-based search in PubMed to collect TC-related genetic information. These gene mutations and fusions were found in particular subtypes of TC (Fig. 1). Papillary thyroid cancer (PTC) and anaplastic thyroid cancer (ATC) had the most related gene mutations. The literature reported that BRAF, RAS, TERT, ETV6, EIF1AX, GNAS, PIK3CA, TP53 and NTRK1 mutations, as well as RET and ALK fusions, were found in PTC. BRAF, TERT, ALK fusion, GNAS, AKT1, PIK3CA, TP53 and PTEN were found in ATC. Fewer gene mutations were found in medullary thyroid cancer (MTC) and follicular thyroid cancer (FTC). RAS, TERT, TSHR, GNAS, PENT and TP53 were found in FTC, while only RET and RAS mutations were found in MTC. The workflow of the NGS mutation analysis is shown in Fig. 1.

Demographic and nodule characteristics. A total of 98 patients with thyroid nodules who had undergone US and pathological examination were included (Table I). DNA samples were taken from FFPE tissue and amplified for sequencing. Based on the 

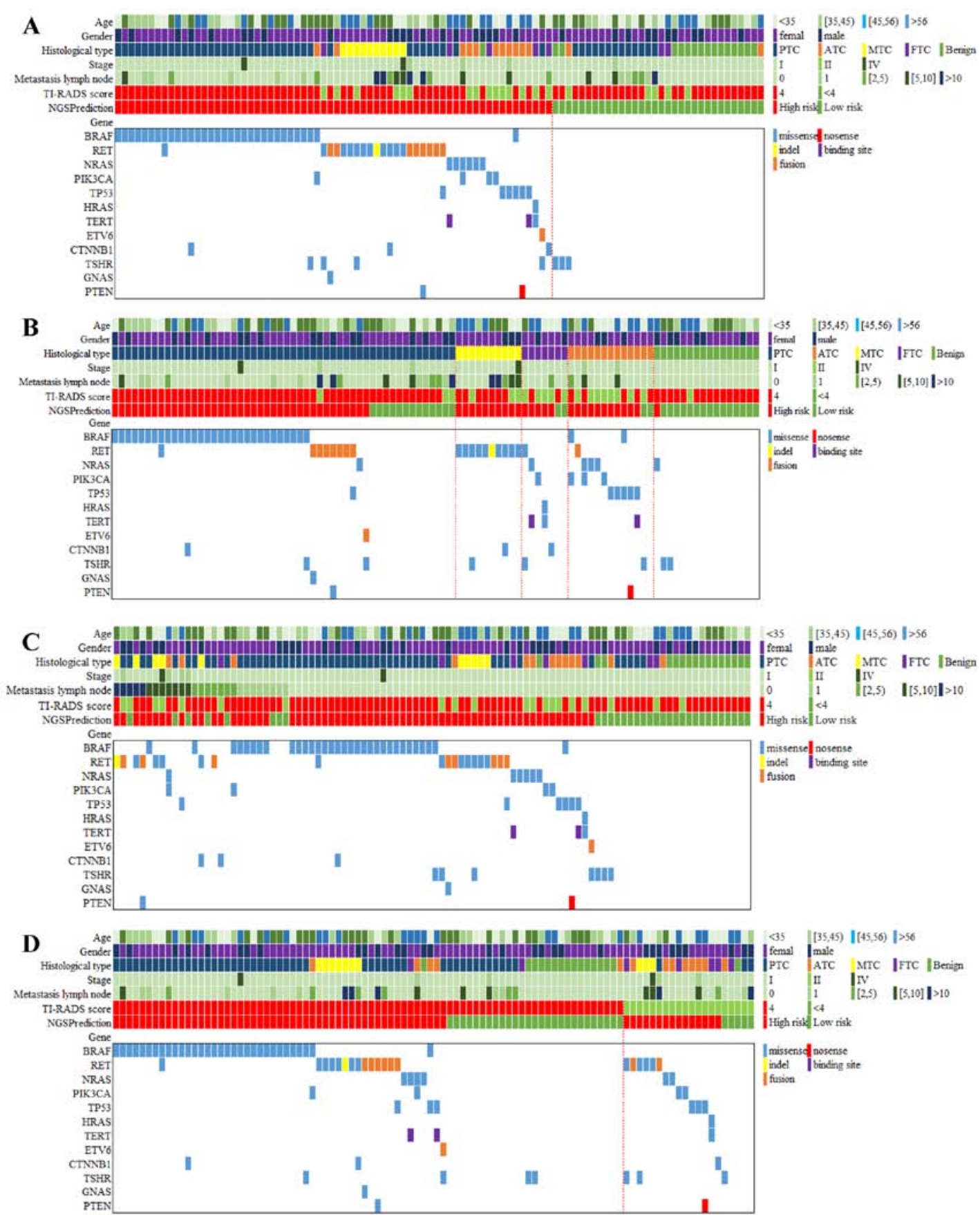

Figure 2. Gene spectrum grouped by benign and malignant thyroid nodules, pathological subtypes, metastatic lymph nodes and TI-RADS. (A) The gene spectrum of the 98 patients contained age, sex, histological diagnosis of cancer type, tumor stage, metastasis lymph node number, TI-RADS category, NGS prediction result and detailed genetic information. (B) The gene spectra of the four TC subtypes varied. For PTC, BRAF mutations were predominant, while RET mutations were detected among all the MTC. A wide range of mutations were positive but none of them showed dominance in ATC and FTC. (C) A total of 27 patients had 1 or more metastasis lymph nodes, 19 of whom carried 23 mutations, including BRAF $(n=8), \operatorname{RET}(n=8)$, NRAS $(n=1)$, PIK3CA ( $n=2)$, TP53 $(\mathrm{n}=1)$, CTNNB1 $(\mathrm{n}=2)$ and PTEN (n=1). (D) Among the 78 US-predicted high-risk patients, 63 cases were diagnosed with TC by histological examination. Out of the 20 US-predicted low-risk patients, only 1 case was diagnosed with benign thyroid nodule by histological examination. TI-RADS, Thyroid Imaging Reporting and Data System; NGS, next-generation sequencing; TC, thyroid cancer; PTC, papillary thyroid cancer; MTC, medullary thyroid cancer; ATC, anaplastic thyroid cancer; FTC, follicular thyroid cancer; US, ultrasound.

histological diagnosis, the demographic data of these patients were separately presented in two groups: benign (16 patients) and malignant ( 82 patients). Age and sex ratio (M/F) in the two groups were $48.00 \pm 6.07$ years and 6/10, and $45.95 \pm 3.20$ years and 25/57, respectively. Characteristics of the nodules, including number, size and various US features (echogenicity, blood flow, microcalcification, aspect ratio and boundary) were collected. Fifteen patients in the benign group and 63 patients in the malignant group were assigned to TI-RADS category 4. The 82 TC patients included 52 PTC, 13 FTC, 10 MTC and 7 ATC cases. Of these, 27 patients were also pathologically diagnosed with lymph node metastasis.

Gene spectrum between benign and malignant thyroid nodules. The gene spectrum of the 98 patients is presented in Fig. 2. In general, 86 mutations of all types of target genes were detected 
in 69 patients ( $70.4 \%$ of 98 patients). Thyroline reported 66 cases $(67.3 \%)$ in high risk, slightly lower than the number of cases with gene mutation. A total of 16 patients (16.3\%) simultaneously carried two gene mutations and no one had $>3$ different gene mutations.

The majority of mutations were missense (73 cases, $84.8 \%$ ), while other mutation types included nonsense (1 case, $1.0 \%$ ), indel (1 case, $1.0 \%$ ), binding site ( 2 cases, $2.0 \%$ ) and fusion ( 9 cases, $9.2 \%$ ). In addition to those cases confirmed to be malignant, 16 patients were diagnosed with benign thyroid nodules through pathological examination. Of these, 3 patients (18.75\%) were positive for gene mutations, including NRAS $(n=1)$ and TSHR $(n=2)$. None of the 16 patients carried $\geq 2$ mutations. Missense mutations were the only mutation type for both NRAS and TSHR.

Gene spectrum in different pathological subtypes of TC. The gene spectra of the four TC subtypes varied. For PTC, BRAF mutations were predominant, while RET mutations were detected among all the MTC cases. A wide range of mutations were positive but none of them showed dominance in ATC or FTC (Fig. 2). Among the 52 PTC patients, 39 (75\%) patients were identified with mutations, an indication for high risk in NGS prediction. Specifically, BRAF mutations occurred in 30 patients $(57.69 \%)$, followed by $\operatorname{RET}(n=8)$, TSHR $(n=2)$, NRAS $(n=1), \operatorname{TP53}(n=1), \operatorname{ETV} 6(n=1)$, CTNNB1 $(n=1)$, GNAS $(n=1)$ and PTEN $(n=1)$. Seven patients $(13.46 \%)$ had multiple mutations, including BRAF/CTNNB1, BRAF/RET, BRAF/TSHR, RET/GNAS, RET/PTEN, RET/TP53 and ETV6/TSHR (Fig. 2). Missense mutations were the most common type of mutation (38 of 46 mutations), detected in all BRAF, NRAS, TP53, CTNNB1, TSHR, GNAS and PTEN genes, while fusion was only observed in RET and ETV6 (8 of 46 mutations). Ten patients were confirmed to be MTC by histological test and all of them were positive for gene mutations, including only RET $(n=10)$, CTNNB1 $(n=1)$ and TSHR $(n=1)$. Other mutations were negative among MTC patients. Two patients $(20 \%)$ exhibited multiple mutations, RET/CTNNB1 $(n=1)$ and RET/TSHR $(n=1)$. Missense mutations still accounted for the most mutations (11 of 12 overall mutations) and just 1 case was detected to be an indel mutation. FTC was diagnosed in 7 patients, among whom $5(71.4 \%)$ were detected with gene mutations. A wide range of mutations were positive, including RET $(n=1)$, NRAS $(n=1)$, PIK3CA $(n=1)$, HRAS $(n=1)$, TERT $(n=2)$, CTNNB1 $(n=1)$ and TSHR $(n=1)$. Three patients $(23.1 \%)$ had multiple mutations, NRAS/TERT, RET/TSHR and HRAS/TERT. Missense mutation was also the most common ( 7 of 8 overall mutations) mutation pattern, and only 1 TERT mutation was reported to occur in the DNA-binding site. A total of 13 patients were diagnosed with ATC, 12 of whom (92.31\%) had gene mutations in BRAF (n=2), RET ( $n=1), \operatorname{NRAS}(n=3), \operatorname{PIK} 3 \mathrm{CA}(\mathrm{n}=3)$, TP53 $(n=5)$, TERT $(n=1)$, TSHR $(n=1)$ and PTEN $(n=1)$. Five patients had multiple mutations, such as BRAF/PIK3CA, NRAS/PIK3CA, BRAF/TP53, TP53/PTEN and TP53/TERT. The greatest diversity in mutation patterns occurred in ATC patients. Missense mutations were again predominant (14 of 17 overall mutations). There was also 1 case of RET fusion mutation, 1 case of TERT binding site mutation, and 1 PTEN nonsense mutation.
Table II. Comparison of the prediction value of NGS and US for clinical TC diagnosis.

\begin{tabular}{lcccc}
\hline A, NGS & & & & \\
\hline $\begin{array}{l}\text { Pathological } \\
\text { diagnosis }\end{array}$ & High risk & Low risk & $\begin{array}{c}\text { Sensitivity } \\
(\%)\end{array}$ & $\begin{array}{c}\text { Specificity } \\
(\%)\end{array}$ \\
\hline $\begin{array}{l}\text { Malignant } \\
\text { Benign }\end{array}$ & 65 & 17 & 79.27 & \\
PPV & 1 & 15 & & 93.75 \\
NPV & $98.48 \%$ & & & \\
\hline Histological & & $46.88 \%$ & & \\
type & Count & Malignant & Benign & $(\%)$ \\
\hline PTC & 53 & 39 & 13 & 75.00 \\
ATC & 13 & 11 & 2 & 84.62 \\
MTC & 10 & 10 & 0 & 100.00 \\
FTC & 7 & 5 & 2 & 71.43 \\
\hline
\end{tabular}

\section{B, US}

\begin{tabular}{lcccc}
\hline $\begin{array}{l}\text { Pathological } \\
\text { diagnosis }\end{array}$ & 4 & $<4$ & $\begin{array}{c}\text { Sensitivity } \\
(\%)\end{array}$ & $\begin{array}{c}\text { Specificity } \\
(\%)\end{array}$ \\
\hline $\begin{array}{l}\text { Malignant } \\
\text { Benign }\end{array}$ & 63 & 19 & 76.83 & \\
PPV & 15 & 1 & & 6.25 \\
NPV & $80.77 \%$ & & & \\
\hline
\end{tabular}

\begin{tabular}{lcrcc}
\hline $\begin{array}{l}\text { Histological } \\
\text { type }\end{array}$ & Count & 4 & $<4$ & $\begin{array}{c}\text { Sensitivity } \\
(\%)\end{array}$ \\
\hline PTC & 52 & 49 & 3 & 94.23 \\
ATC & 13 & 5 & 8 & 38.46 \\
MTC & 10 & 7 & 3 & 70.00 \\
FTC & 7 & 2 & 5 & 28.57 \\
\hline
\end{tabular}

(A) Among the NGS high-risk patients, 65 were confirmed to be malignant, with only 1 benign sample. In the low-risk patients, 15 were benign and 17 were malignant. The sensitivity and specificity of NGS were 79.27 and $93.75 \%$, respectively. The PPV and NPV were 98.48 and $46.88 \%$, respectively. The sensitivity of NGS was $>70 \%$ in all subtypes, particularly in MTC, when it was up to $100 \%$. (B) Among the high-risk patients by US examination, 63 were confirmed to be malignant and 15 were benign. In the low-risk patients, 1 was benign and 19 were malignant. The sensitivity and specificity of US were 76.83 and $6.25 \%$, respectively. The PPV and NPV were 80.77 and $5.00 \%$, respectively. As analyzed by pathological subtypes, US had the highest sensitivity in PTC, up to $94.23 \%$. However, the sensitivity in ATC and FTC was only 38.46 and $28.57 \%$, respectively. NGS, next-generation sequencing; US, ultrasound; TC, thyroid cancer; PPV, positive predictive value; NPV, negative predictive value; PTC, papillary thyroid cancer; ATC, anaplastic thyroid cancer; MTC, medullary thyroid cancer; FTC, follicular thyroid cancer.

Greater prediction value for NGS compared to US. The overall predictive abilities for thyroid nodule malignancy 

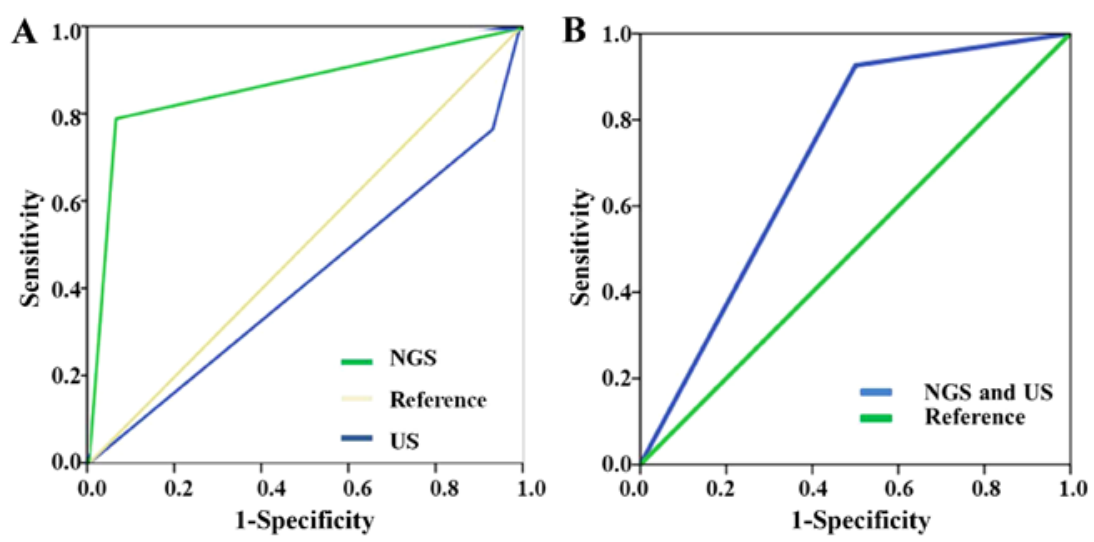

Figure 3. ROCs of NGS and US. (A) The AUC of NGS was 0.865, while the AUC of US was 0.415. (B) In situations where NGS and US drew the same conclusion, the sensitivity of combined NGS and US was $92.6 \%$ and the specificity was $50 \%$. The AUC of combined NGS and US was 0.712 . ROC, receiver operator characteristic; NGS, next-generation sequencing; US, ultrasound; AUC, area under curve.

by NGS and US are presented in Table II. Among the NGS high-risk patients, 65 were confirmed to be malignant, with only 1 benign sample. All patients with multiple mutations were malignant and reported with high risk. In the low-risk patients, 15 were benign and 17 were malignant. Thus, the sensitivity and specificity of NGS prediction were 79.27 and $93.75 \%$, respectively. The PPV and NPV for NGS were 98.48 and $46.88 \%$, respectively. Among the high-risk patients identified by US examination, 63 were confirmed to be malignant and 15 were benign. In the low-risk patients, 1 was benign and 19 were malignant. Thus, the sensitivity and specificity of US examination were 76.83 and $6.25 \%$, respectively. The PPV and NPV were 80.77 and $5.00 \%$, respectively.

In terms of different pathological subtypes, the sensitivity of NGS was consistently $>70 \%$, particularly in MTC, when it was up to $100 \%$. US had the highest sensitivity in PTC, up to $94.23 \%$. The panel reported all the cases $(100 \%)$ to be at high risk, while only 7 cases $(70 \%)$ were defined as high risk based on US. However, the sensitivity in ATC and FTC was only 38.46 and $28.57 \%$, respectively. A total of 11 ATC patients $(84.62 \%)$ were reported as having high risk through NGS test, slightly lower than the number of patients with mutations. However, US only found 5 ATC cases to be at high risk. The panel reported 5 in 7 FTC cases (71.43\%) to be high risk, and only 2 cases $(28.57 \%)$ were defined as high risk through US. The ROCs of NGS and US are presented in Fig. 3. The area under curve (AUC) of NGS test was 0.865 , while the AUC of US test was 0.415 . The prediction value of NGS was higher than that of US. In situations where NGS and US drew the same conclusion, the sensitivity of combined NGS and US was $92.6 \%$ and the specificity was $50 \%$. The AUC of combined NGS and US was 0.712.

Differential profile among lymph node metastasis degrees of thyroid nodules. By examining the lymph nodes of all patients with thyroid nodules, 27 patients had $\geq 1$ metastasis lymph nodes, including PTC ( $\mathrm{n}=18)$, MTC $(\mathrm{n}=5)$, FTC $(\mathrm{n}=1)$ and ATC $(\mathrm{n}=3)$ patients. Specifically, $5(18.52 \%)$ patients had $>10$ metastasis lymph nodes, 7 (25.93\%) had 5-10 nodes, 7 (25.93\%) had $2-5$ nodes and $8(29.63 \%)$ had 1 node. Patients with $>1$ metastatic lymph node tended to show a greater diversity in cancer subtype, while all patients with only 1 metastasis lymph node were diagnosed with PTC. A total of 8 patients (29.63\%) with metastasis lymph nodes were detected with no gene mutation by NGS and reported to be at low risk as well. The remaining patients had a variety of mutations that differed in both gene and mutation pattern. Nineteen patients carried 23 mutations, including BRAF $(\mathrm{n}=8)$, RET $(\mathrm{n}=8)$, NRAS $(\mathrm{n}=1)$, PIK3CA $(\mathrm{n}=2)$, TP53 $(\mathrm{n}=1)$, CTNNB1 $(\mathrm{n}=2)$ and PTEN $(\mathrm{n}=1)$. However, no correlation was found between the variant allele frequency of BRAF, NRAS, RET mutation, RET fusion and number of metastatic lymph nodes (Fig. 4; $\mathrm{P}>0.05$ ).

Prediction difference for the high-risk thyroid nodules between $N G S$ and US. There was inconsistency in high-risk identification between NGS and US (Fig. 2). Among the 78 US-predicted high-risk patients, $51(65.38 \%)$ were reported as high risk by NGS. Among 20 US-predicted low-risk patients, only 5 (25\%) were reported as low risk by NGS. We picked out two patients (case 1 and 2) diagnosed with benign thyroid nodule by ultrasonic diagnosis but assessed as malignant by pathological results (Fig. 5). US showed no typical features such as hypoechogenicity, unclear margins, rich blood flow, microcalcification or taller-than-wide shape. However, both cases were PTC with BRAF mutation. Simultaneously, we also selected two other patients (case 3 and 4) with consistent results among NGS, US and pathological diagnosis. For these cases, US demonstrated typically malignant features, including microcalcification and hypoechogenicity (Fig. 5). Consistently, NGS also detected TC-related driver mutation, such as NRAS and TERT in case 3 , and TP53 and TERT in case 4.

\section{Discussion}

A complete understanding of the molecular mechanisms of tumor formation is essential for providing accurate diagnoses and personalized treatments. In the past, single gene assays have been commonly used for finding molecular alterations in TC. TC harbors characteristic genetic alterations, including point mutations for proto-oncogenes (BRAF and RAS) and chromosomal rearrangements (RET), which vary with histological subtypes $(12,16)$. Presently, NGS technology can simultaneously analyze hundreds of genes of interest using targeted sequencing panels, indicating 

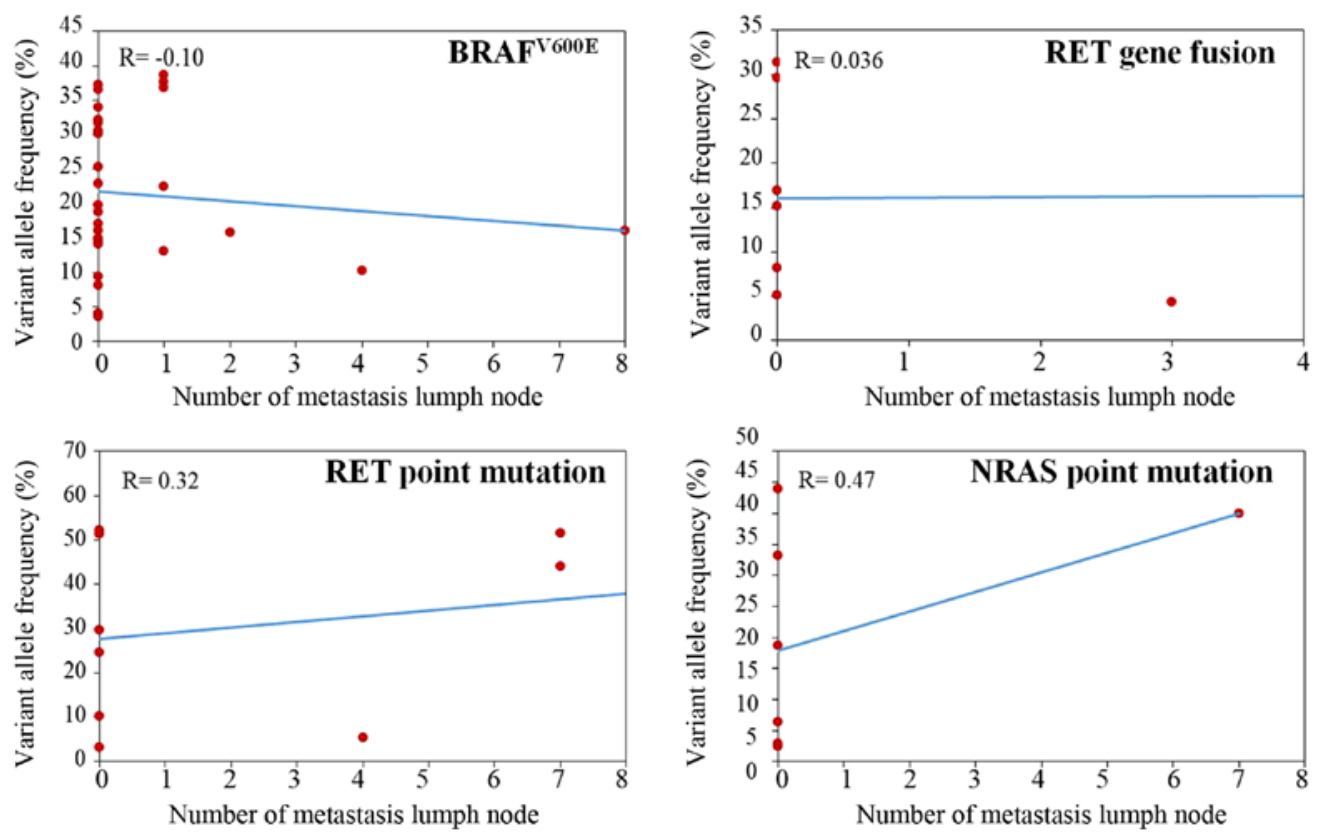

Figure 4. Correlation between variant allele frequency and number of metastatic lymph nodes. Spearman's analysis was performed for the variant allele frequency of BRAF, NRAS and RET mutation, RET fusion and number of metastatic lymph nodes. As a result, no correlation was found between the variant allele frequency of BRAF, NRAS and RET mutation, RET fusion and number of metastatic lymph nodes. $\mathrm{P}>0.05$.
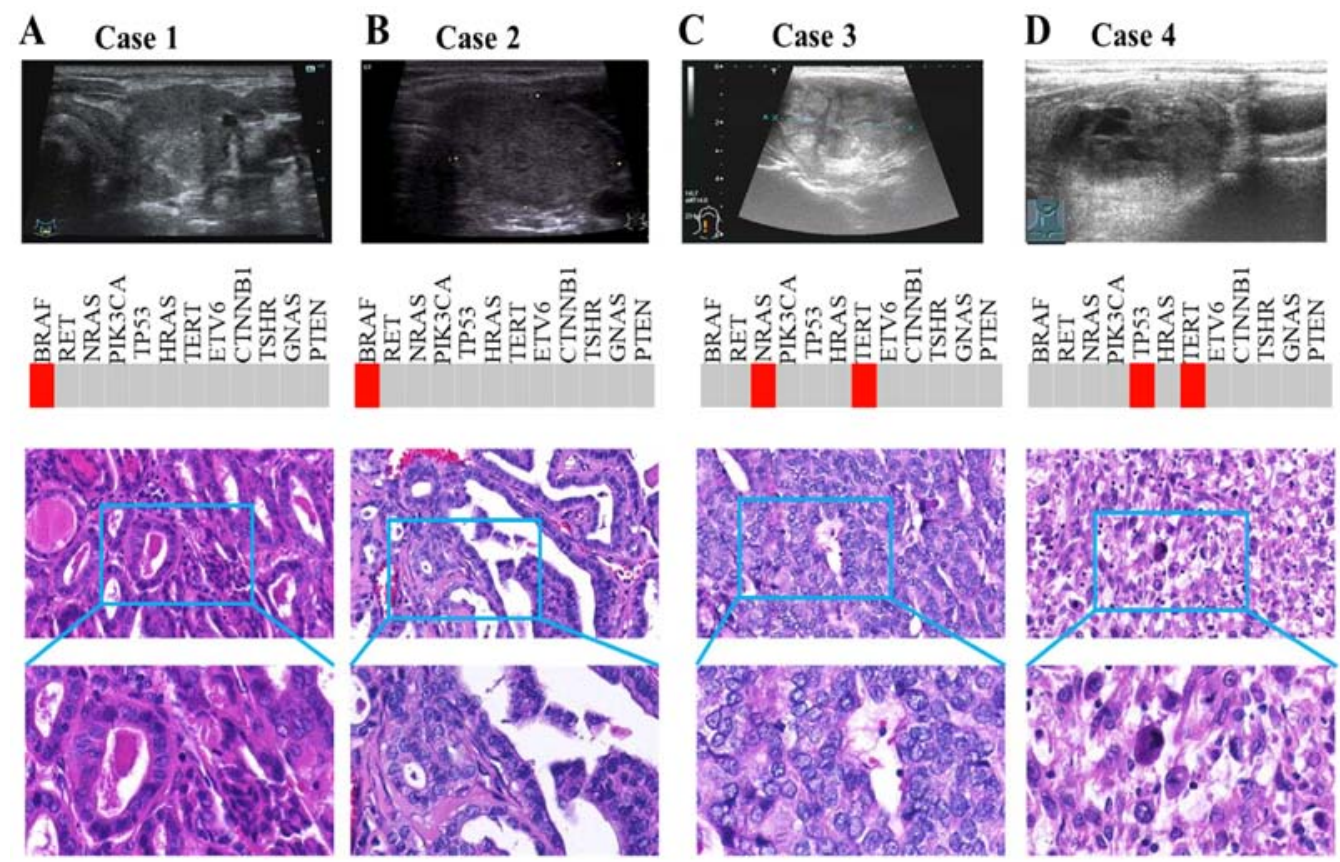

Figure 5. Prediction difference between NGS and US for high-risk thyroid nodules. (A and B) Case 1 and 2 were diagnosed via US with benign thyroid nodule, but pathological results revealed malignancy. US showed no typical features. However, both cases were PTC with BRAF mutation. (C and D) Case 3 and 4 had consistent results among NGS, US and pathological diagnosis. US demonstrated typically malignant features, including microcalcification and hypoechogenicity. Consistently, NGS also detected TC-related driver mutations, such as NRAS and TERT in case 3, and TP53 and TERT in case 4. NGS, next-generation sequencing; US, ultrasound; PTC, papillary thyroid cancer; TC, thyroid cancer.

that NGS-based molecular tests for oncology research and clinical practice are rapidly evolving (17). NGS allows for simultaneous high-throughput sequencing analysis of variable genetic alterations and provides a comprehensive understanding of tumor biology, which can improve diagnostic accuracy and is useful for providing personalized treatments for TC patients.
Published literature increasingly indicates that gene mutations can be specific biomarkers for clinical TC diagnosis $(13,16,18)$. As the most common gene mutation of TC, BRAF mutations were found in $\sim 45-60 \%$ of PTC cases $(12,19)$. RAS mutations (HRAS, KRAS and NRAS) were found in $40-53 \%$ of FTC cases $(20,21)$. RET was commonly found to be mutated in both familial and sporadic MTC (22). In our 
study, analysis of patients with malignant nodules showed great heterogeneity in gene and mutation patterns between different TCs. BRAF mutations were detected in $57.69 \%$ of PTC, while RAS mutations were only found in $28.57 \%(2 / 7)$ of FTC cases. A previous study including 35 FTCs found that 16 patients (45.7\%) harbored RAS mutations (23). Compared with this, the low positive rate of RAS in the current study may be attributed to the recruited sample size (7 FTC patients). Interestingly, in the FTC subgroup, two patients had either NRAS/TERT or HRAS/TERT multiple mutation patterns. TERT promoter mutations are more prevalent in advanced TCs, particularly those harboring BRAF and RAS mutations $(24,25)$. In addition, RET mutations were detected in all MTC patients. Specifically, RET mutations may suggest a strong genotype-phenotype correlation with MTC (26), indicating that it may be a reliable biomarker for MTC diagnosis.

In addition to well-known BRAF, RAS and RET mutations, NGS technology facilitated detection of new somatic alterations in TC, the significance of which has not yet been explored in detail. We found many gene mutations in particular tumor subtypes that are seldom reported, such as CTNNB1 and TSHR in PTC; NRAS and TSHR in ATC; CTNNB1 in MTC; and PIK3CA and CTNNB1 in FTC. Interestingly, TSHR was traditionally reported in FTC (27), but found in all subtypes by our gene spectrum. Thus, we hypothesize that TSHR may be a common driver gene for TC. Due to sample size limitations, our gene spectrum results may vary from previous studies (27-29), and some mutations in our panel were negative among TC patients.

US is the preferred non-invasive diagnostic method recommended by current American Thyroid Association guidelines (8). Nonetheless, the diagnosis value of TI-RADS is controversial. A prospective study including $>2,000$ patients reported that the diagnostic accuracy of US of $\geq 1$ malignant features was only $72.6 \%$ (9). In our study, we focused on the application of NGS for predicting biological characteristics of thyroid nodules, including a novel comparison of the predictive efficacy between NGS and US. Both NGS and US demonstrated a high sensitivity in diagnosis of TC, but both showed inconsistency in high-risk identification. TC patients with BRAF were all classified as high risk by US. However, for those without BRAF mutations, prediction accuracy dropped markedly. BRAF mutations were mainly detected in PTC $(12,19)$, and the US features varied between subtypes $(30,31)$. The clinical application of US-based TI-RADS may be confined to PTC, which may lead to missed and delayed diagnosis of other subtypes. The results suggest that NGS technology in differentiation of benign and malignancy possess more advantages in terms of sensitivity, specificity, PPV and NPV compared to US. The sensitivity and specificity of combined NGS and US were superior to US alone. Therefore, NGS in combination with US may have better diagnostic and/or prognostic value for TC patients.

Another purpose of the panel was to investigate its ability to reflect lymph node metastasis status. Previous studies found that $19.4-84 \%$ of TC patients have lymph node metastases $(32,33), 33 \%$ of which are missed by preoperative US examination $(7,34)$. Patients with lymph node metastasis often need more aggressive therapy, including radiative iodine treatment and more frequent follow-up (8). Based on histological examination in the current study, 27 patients had $\geq 1$ metastatic lymph nodes. Mutations in genes including BRAF, RET, NRAS, PIK3CA, TP53, CTNNB1 and PTEN were found in 19 patients. These mutations may be a key driving factor for lymph node metastasis. For deeper insight into the relationship between the abundance of mutant allele and metastasis status, we selected BRAF, RET and NRAS mutations as well as RET fusion for correlation analysis. However, we did not find a significant correlation between the variant allele frequency of aforementioned gene mutations and the number of metastatic lymph nodes. This finding may have been due to the small sample size. Therefore, the impact of genetics on lymph node metastasis has yet to be determined.

The association of BRAF mutation with more aggressive clinicopathological features and poorer outcomes has been under debate for some time. Certain studies have demonstrated a role of BRAF in tumor aggressiveness in PTC $(35,36)$, but others have failed to do so $(37,38)$. A new genetic alteration, the point mutation in the TERT promoter, was recently described in TC and has been shown to be associated with increased aggressiveness and poorer clinical prognosis $(39,40)$. Notably, Xing et al found that PTC patients with coexisting BRAF and TERT mutations had the worst clinicopathological outcomes (41). TERT mutations were found in $12.9 \%(21 / 163)$ of Portuguese PTC patients (42). However, we did not find TERT mutations in PTC patients, or coexisting BRAF and TERT mutations. TERT mutation was found in $4.4 \%(20 / 455)$ and $4.1 \%$ (27/653) PTC patients in another two Chinese studies, respectively $(43,44)$. Additionally, the prevalence $(14.3 \%, 1 / 7)$ of TERT promoter mutation in ATC patients from our study was significantly $<46.3 \%(25 / 54)$ and $38.7 \%$ (41/106) from Liu et al and Shi et al respectively $(39,45)$. TERT promoter mutations may play an important role in distant metastases of TC (42). However, no ATC patients in our cohort presented distant metastases. Thus, the reason why the mutation rate of TERT promoters was low in our detection platform may be attributed to the small sample size of ATC. On the other hand, it may be related with the heterogeneity of ATC biological behavior.

In conclusion, NGS exhibits advantages in discriminating benign and malignant thyroid nodules compared with traditional US-based TI-RADS, simultaneously providing insight into the pathological subtypes of TC. The novel use of NGS-based Thyroline in conjunction with US may pave the way for increasingly accurate and timely diagnoses of TC compared to conventional methods. However, the current findings may assist the application of NGS in FNA samples. A limitation of our analysis was that it was performed in FFPE tissues, not in FNA samples. In the future, the combined evaluation of target-medicine-related gene status included in the Thyroline panel may be used to assist precision treatment for TC patients, leading to improved clinical outcomes.

\section{Acknowledgements}

Not applicable.

\section{Funding}

The present study was supported by the National Natural Science Foundation of China (81272932, 30900650, 81372501, 
81572260), the Natural Science Foundation of Guangdong Province (S2012040007756, S201281572260010008378, S2013010015327, 2013B021800126, 2015A020214010, 2016A020215055 and 2013B021800259).

\section{Availability of data and materials}

The datasets used and/or analyzed during the current study are available from the corresponding author on reasonable request.

\section{Authors' contributions}

ZK, YL, YZ and HX conceived and designed the study. ZK, LC and CS performed the histological examination. JLiang was a contributor to the US examination. SP and BL were contributors in the data statistics. ZK and YL wrote the report. JLi, MK, LS, SY, JC and WL reviewed and edited the manuscript and were also involved in the conception of the study. All authors read and approved the final manuscript. All authors agreed to be accountable for all aspects of the work in ensuring that questions related to the accuracy or integrity of any part of the work are appropriately investigated and resolved.

\section{Ethics approval and consent to participate}

The study had got the ethics approval of the Ethics Committee of the First Affiliated Hospital of Sun Yat-sen University and the reference number was 2017224.

\section{Patient consent for publication}

Not applicable.

\section{Competing interests}

The authors declare that they have no competing interests.

\section{References}

1. Kilfoy BA, Zheng T, Holford TR, Han X, Ward MH, Sjodin A Zhang Y, Bai Y, Zhu C, Guo GL, et al: International patterns and trends in thyroid cancer incidence, 1973-2002. Cancer Causes Control 20: 525-531, 2009.

2. Rego-Iraeta A, Perez-Mendez LF, Mantinan B and Garcia-Mayor RV: Time trends for thyroid cancer in northwestern Spain: True rise in the incidence of micro and larger forms of papillary thyroid carcinoma. Thyroid 19: 333-340, 2009.

3. Dal Maso L, Lise M, Zambon P, Falcini F, Crocetti E, Serraino D, Cirilli C, Zanetti R, Vercelli M, Ferretti S, et al; AIRTUM Working Group: Incidence of thyroid cancer in Italy, 1991-2005: Time trends and age-period-cohort effects. Ann Oncol 22: 957-963, 2011.

4. Haggar FA, Preen DB, Pereira G, Holman CD and Einarsdottir K: Cancer incidence and mortality trends in Australian adolescents and young adults, 1982-2007. BMC Cancer 12: 151, 2012.

5. Ellison LF and Wilkins K: Canadian trends in cancer prevalence. Health Rep 23: 7-16, 2012.

6. Gharib H, Papini E, Garber JR, Duick DS, Harrell RM, Hegedüs L, Paschke R, Valcavi R and Vitti P; AACE/ACE/AME Task Force on Thyroid Nodules: American Association of Clinical Endocrinologists, American College of Endocrinology, and Associazione Medici Endocrinologi medical guidelines for clinical practice for the diagnosis and management of thyroid nodules - 2016 Update. Endocr Pract 22: 622-639, 2016.
7. Moo TA, McGill J, Allendorf J, Lee J, Fahey T III and Zarnegar R: Impact of prophylactic central neck lymph node dissection on early recurrence in papillary thyroid carcinoma. World J Surg 34: 1187-1191, 2010.

8. Haugen BR, Alexander EK, Bible KC, Doherty GM, Mandel SJ, Nikiforov YE, Pacini F, Randolph GW, Sawka AM, Schlumberger M, et al: 2015 American Thyroid Association management guidelines for adult patients with thyroid nodules and differentiated thyroid cancer: The American Thyroid Association Guidelines Task Force on thyroid nodules and differentiated thyroid cancer. Thyroid 26: 1-133, 2016.

9. Kim SJ, Moon WK and Cho N: Sonographic criteria for fine-needle aspiration cytology in a Korean female population undergoing thyroid ultrasound screening. Acta Radiol 51: 475-481, 2010.

10. Nikiforov YE, Carty SE, Chiosea SI, Coyne C, Duvvuri U, Ferris RL, Gooding WE, Hodak SP, LeBeau SO, Ohori NP, et al: Highly accurate diagnosis of cancer in thyroid nodules with follicular neoplasm/suspicious for a follicular neoplasm cytology by ThyroSeq v2 next-generation sequencing assay. Cancer 120 : 3627-3634, 2014.

11. Suh YJ, Son EJ, Moon HJ, Kim EK, Han KH and Kwak JY: Utility of thyroglobulin measurements in fine-needle aspirates of space occupying lesions in the thyroid bed after thyroid cancer operations. Thyroid 23: 280-288, 2013.

12. Nikiforova MN, Wald AI, Roy S, Durso MB and Nikiforov YE: Targeted next-generation sequencing panel (ThyroSeq) for detection of mutations in thyroid cancer. J Clin Endocrinol Metab 98: E1852-E1860, 2013.

13. Hsiao SJ and Nikiforov YE: Molecular approaches to thyroid cancer diagnosis. Endocr Relat Cancer 21: T301-T313, 2014.

14. Nikiforov YE, Ohori NP, Hodak SP, Carty SE, LeBeau SO, Ferris RL, Yip L, Seethala RR, Tublin ME, Stang MT, et al: Impact of mutational testing on the diagnosis and management of patients with cytologically indeterminate thyroid nodules: A prospective analysis of 1056 FNA samples. J Clin Endocrinol Metab 96: 3390-3397, 2011.

15. Kwak JY, Han KH, Yoon JH, Moon HJ, Son EJ, Park SH, Jung HK, Choi JS, Kim BM and Kim EK: Thyroid imaging reporting and data system for US features of nodules: A step in establishing better stratification of cancer risk. Radiology 260: 892-899, 2011.

16. Cha YJ and Koo JS: Next-generation sequencing in thyroid cancer. J Transl Med 14: 322, 2016.

17. LeBlanc VG and Marra MA: Next-generation sequencing approaches in cancer: Where have they brought us and where will they take us? Cancers (Basel) 7: 1925-1958, 2015.

18. Nikiforov YE and Nikiforova MN: Molecular genetics and diagnosis of thyroid cancer. Nat Rev Endocrinol 7: 569-580, 2011.

19. Chiosea S, Nikiforova M, Zuo H, Ogilvie J, Gandhi M, Seethala RR, Ohori NP and Nikiforov Y: A novel complex BRAF mutation detected in a solid variant of papillary thyroid carcinoma. Endocr Pathol 20: 122-126, 2009.

20. Lemoine NR, Mayall ES, Wyllie FS, Williams ED, Goyns M, Stringer B and Wynford-Thomas D: High frequency of ras oncogene activation in all stages of human thyroid tumorigenesis. Oncogene 4: 159-164, 1989.

21. Motoi N, Sakamoto A, Yamochi T, Horiuchi H, Motoi T and Machinami R: Role of ras mutation in the progression of thyroid carcinoma of follicular epithelial origin. Pathol Res Pract 196: $1-7,2000$.

22. Kloos RT, Eng C, Evans DB, Francis GL, Gagel RF, Gharib H, Moley JF, Pacini F, Ringel MD, Schlumberger M, et al; American Thyroid Association Guidelines Task Force: Medullary thyroid cancer: Management guidelines of the American Thyroid Association. Thyroid 19: 565-612, 2009.

23. Jeong SH, Hong HS, Kwak JJ and Lee EH: Analysis of RAS mutation and PAX8/PPAR $\gamma$ rearrangements in follicular-derived thyroid neoplasms in a Korean population: Frequency and ultrasound findings. J Endocrinol Invest 38: 849-857, 2015.

24. Song YS, Lim JA, Choi H, Won JK, Moon JH, Cho SW, Lee KE, Park YJ, Yi KH, Park DJ, et al: Prognostic effects of TERT promoter mutations are enhanced by coexistence with BRAF or RAS mutations and strengthen the risk prediction by the ATA or TNM staging system in differentiated thyroid cancer patients. Cancer 122: 1370-1379, 2016

25. Landa I, Ganly I, Chan TA, Mitsutake N, Matsuse M, Ibrahimpasic T, Ghossein RA and Fagin JA: Frequent somatic TERT promoter mutations in thyroid cancer: Higher prevalence in advanced forms of the disease. J Clin Endocrinol Metab 98: E1562-E1566, 2013. 
26. Frank-Raue K, Rondot S and Raue F: Molecular genetics and phenomics of RET mutations: Impact on prognosis of MTC. Mol Cell Endocrinol 322: 2-7, 2010.

27. Lado-Abeal J, Celestino R, Bravo SB, Garcia-Rendueles ME, de la Calzada J, Castro I, Castro P, Espadinha C, Palos F, Soares $\mathrm{P}$, et al: Identification of a paired box gene 8-peroxisome proliferator-activated receptor $\gamma$ (PAX8-PPAR $\gamma$ ) rearrangement mosaicism in a patient with an autonomous functioning follicular thyroid carcinoma bearing an activating mutation in the TSH receptor. Endocr Relat Cancer 17: 599-610, 2010.

28. Kim SH, Kang JG, Kim CS, Ihm SH, Choi MG, Yoo HJ and Lee SJ: 17-Allylamino-17-demethoxygeldanamycin and herbimycin $\mathrm{A}$ induce cell death by modulating $\beta$-catenin and PI3K/AKT signaling in FRO anaplastic thyroid carcinoma cells. Anticancer Res 35: 5453-5460, 2015.

29. García-Rostán G, Costa AM, Pereira-Castro I, Salvatore G, Hernandez R, Hermsem MJ, Herrero A, Fusco A, CameselleTeijeiro J and Santoro M: Mutation of the PIK3CA gene in anaplastic thyroid cancer. Cancer Res 65: 10199-10207, 2005.

30. Jeh SK, Jung SL, Kim BS and Lee YS: Evaluating the degree of conformity of papillary carcinoma and follicular carcinoma to the reported ultrasonographic findings of malignant thyroid tumor. Korean J Radiol 8: 192-197, 2007.

31. Machens A, Holzhausen HJ and Dralle H: The prognostic value of primary tumor size in papillary and follicular thyroid carcinoma. Cancer 103: 2269-2273, 2005.

32. Lee YC, Shin SY, Kwon KH and Eun YG: Incidence and clinical characteristics of prelaryngeal lymph node metastasis in papillary thyroid cancer. Eur Arch Otorhinolaryngol 270: 2547-2550, 2013

33. Noguchi M, Yamada H, Ohta N, Ishida T, Tajiri K, Fujii H and Miyazaki I: Regional lymph node metastases in well-differentiated thyroid carcinoma. Int Surg 72: 100-103, 1987.

34. Rotstein L: The role of lymphadenectomy in the management of papillary carcinoma of the thyroid. J Surg Oncol 99: 186-188, 2009.

35. Xing M: BRAF mutation in papillary thyroid cancer: Pathogenic role, molecular bases, and clinical implications. Endocr Rev 28: 742-762, 2007.

36. Kim TH, Park YJ, Lim JA, Ahn HY, Lee EK, Lee YJ, Kim KW, Hahn SK, Youn YK, Kim KH, et al: The association of the BRAF(V600E) mutation with prognostic factors and poor clinical outcome in papillary thyroid cancer: A meta-analysis. Cancer 118: 1764-1773, 2012
37. Gouveia C, Can NT, Bostrom A, Grenert JP, van Zante A and Orloff LA: Lack of association of BRAF mutation with negative prognostic indicators in papillary thyroid carcinoma: The University of California, San Francisco, experience. JAMA Otolaryngol Head Neck Surg 139: 1164-1170, 2013.

38. Lee KC, Li C, Schneider EB, Wang Y, Somervell H, Krafft M, Umbricht CB and Zeiger MA: Is BRAF mutation associated with lymph node metastasis in patients with papillary thyroid cancer? Surgery 152: 977-983, 2012.

39. Liu X, Bishop J, Shan Y, Pai S, Liu D, Murugan AK, Sun H, El-Naggar AK and Xing M: Highly prevalent TERT promoter mutations in aggressive thyroid cancers. Endocr Relat Cancer 20: 603-610, 2013.

40. Melo M, da Rocha AG, Vinagre J, Batista R, Peixoto J, Tavares C, Celestino R, Almeida A, Salgado C, Eloy C, et al: TERT promoter mutations are a major indicator of poor outcome in differentiated thyroid carcinomas. J Clin Endocrinol Metab 99: E754-E765, 2014.

41. Xing M, Liu R, Liu X, Murugan AK, Zhu G, Zeiger MA, Pai S and Bishop J: BRAF V600E and TERT promoter mutations cooperatively identify the most aggressive papillary thyroid cancer with highest recurrence. J Clin Oncol 32: 2718-2726, 2014.

42. Melo M, Gaspar da Rocha A, Batista R, Vinagre J, Martins MJ, Costa G, Ribeiro C, Carrilho F, Leite V, Lobo C, et al: TERT, BRAF, and NRAS in primary thyroid cancer and metastatic disease. J Clin Endocrinol Metab 102: 1898-1907, 2017.

43. Sun J, Zhang J, Lu J, Gao J, Ren X, Teng L, Duan H, Lin Y, Li X, Zhang B, et al: BRAF V600E and TERT promoter mutations in papillary thyroid carcinoma in Chinese patients. PLoS One 11: e0153319, 2016

44. Jin L, Chen E, Dong S, Cai Y, Zhang X, Zhou Y, Zeng R, Yang F, Pan C, Liu Y, et al: BRAF and TERT promoter mutations in the aggressiveness of papillary thyroid carcinoma: A study of 653 patients. Oncotarget 7: 18346-18355, 2016.

45. Shi X, Liu R, Qu S, Zhu G, Bishop J, Liu X, Sun H, Shan Z, Wang E, Luo Y, et al: Association of TERT promoter mutation $1,295,228 \mathrm{C}>\mathrm{T}$ with BRAF V600E mutation, older patient age, and distant metastasis in anaplastic thyroid cancer. J Clin Endocrinol Metab 100: E632-E637, 2015.

This work is licensed under a Creative Commons

Attribution-NonCommercial-NoDerivatives 4.0 International (CC BY-NC-ND 4.0) License. 